\title{
Predicting Wave Glider Speed from Environmental Measurements
}

\author{
Ryan N. Smith*, Jnaneshwar Das ${ }^{\dagger}$, Graham Hine ${ }^{\ddagger}$, Will Anderson ${ }^{\ddagger}$ and Gaurav S. Sukhatme ${ }^{\dagger}$ \\ ${ }^{*}$ School of Engineering Systems, Queensland University of Technology, Brisbane, QLD 4000, Australia \\ Email: ryan.smith@qut.edu.au \\ ${ }^{\dagger}$ Robotic Embedded Systems Laboratory, University of Southern California, Los Angeles, CA 90089 \\ Email: jnaneshd,gaurav@usc.edu \\ ${ }^{\ddagger}$ Liquid Robotics, 1329 Moffett Park Drive Sunnyvale, CA 94089, USA \\ Email: graham.hine,will.anderson@ liquidr.com
}

\begin{abstract}
In the ocean science community, researchers have begun employing novel sensor platforms as integral pieces in oceanographic data collection, which have significantly advanced the study and prediction of complex and dynamic ocean phenomena. These innovative tools are able to provide scientists with data at unprecedented spatiotemporal resolutions. This paper focuses on the newly developed Wave Glider platform from Liquid Robotics. This vehicle produces forward motion by harvesting abundant natural energy from ocean waves, and provides a persistent ocean presence for detailed ocean observation. This study is targeted at determining a kinematic model for offline planning that provides an accurate estimation of the vehicle speed for a desired heading and set of environmental parameters. Given the significant wave height, ocean surface and subsurface currents, wind speed and direction, we present the formulation of a system identification to provide the vehicle's speed over a range of possible directions.
\end{abstract}

\section{INTRODUCTION}

Ocean processes are dynamic, complex, and occur on multiple spatial and temporal scales. To obtain a synoptic view of such processes, ocean scientists must collect data over long time periods. This requires extensive time at sea, or an autonomous vehicle that can remain deployed for long durations. By harvesting abundant natural energy from ocean waves, the Liquid Robotics Wave Glider (see Fig. 1) provides a persistent ocean presence for detailed ocean observation and the study of persistent robotic control. With a demonstrated endurance exceeding one year, the Wave Glider (WG) offers a unique platform for ongoing engineering development and a range of new applications for robotics research and ocean scientists alike [1], [2].

The WG is an interesting vehicle from the design, operation and control perspectives. The vehicle is composed of a two-part architecture; a surface component housing the electronics, and a subsurface wing system that generates locomotion. This vehicle provides an interesting path planning problem since it is under-actuated, and although the on-board controller maintains an accurate heading, the speed of the vehicle is entirely environment-dependent. The primary open problem in controlling the WG is predicting its speed, given a desired heading and known or predicted environmental conditions, e.g., significant wave height, wave peak period, wind speed and direction, and the speed and direction of the ocean surface and subsurface currents. The dual to this problem is to design a path that satisfies a speed constraint for the given weather conditions. Sea trials have been conducted,

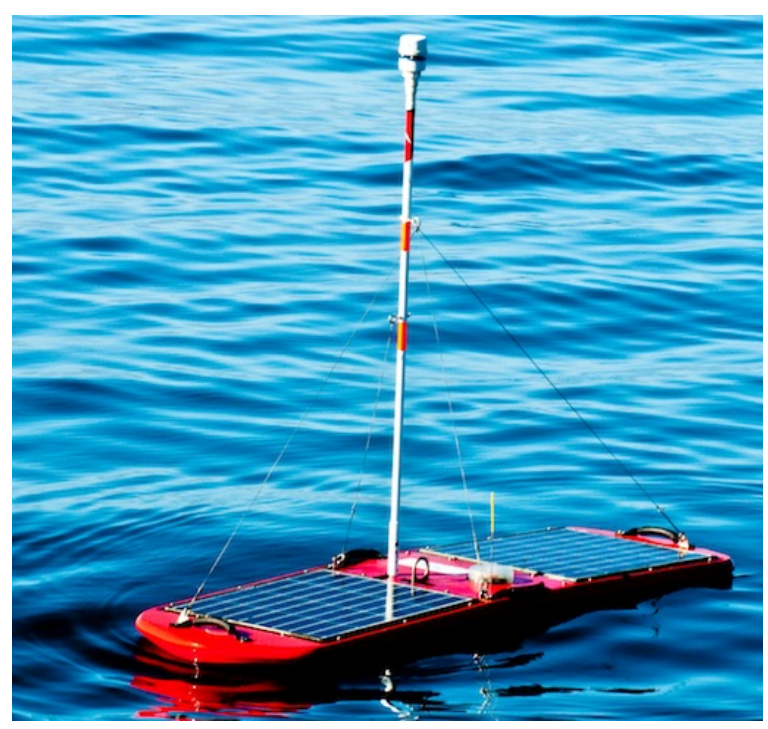

Fig. 1: The Wave Glider platform.

and analysis performed here to create a first-order model to predict the speed of the WG given measured environmental conditions. Examination of this unique platform and developing a detailed model of its motion are rich problems in control theory that will advance the state-of-the-art in applications to marine vehicles.

\section{BACKGROUND AND MOTIVATION}

Currently, the majority of ocean research employs an Eulerian sampling approach, gathering point measurements from fixed moorings, or methodically implementing a regular grid pattern with a research vessel or autonomous robotic vehicle, taking measurements as it moves. Regardless of the method, the region of interest generally remains fixed, and the dynamic features of scientific interest enter and exit this region, moving with the oceanic currents. Research supported through the Monterey Bay Aquarium Research Institute's (MBARI) Controlled, Agile, and Novel Observing Network (CANON) project [3] aims to change this sampling methodology. By coordinating fixed instruments, ships, and robotic vehicles that drift with the currents or actively follow ocean features, targeting and tracking a feature of scientific interest presents an alternative research strategy. Such an endeavour requires merging of observations and model data, 
as well as coordination of a diverse fleet of static and mobile sensor platforms. The vision is to develop the technological innovations necessary to provide a synoptic view of the environment at unprecedented resolution, by letting environmental conditions guide sampling decisions in real time and in situ.

One component that will contribute to the success of this innovative sampling approach is a mobile sensor platform that is able to provide a persistent presence in a given region, or track ocean features for long periods of time. The WG platform precisely fills and extends this role by providing a sensor platform that has extremely high endurance, with the capability to act as a static sensor node, as well as a mobile asset. The utility of the WG was demonstrated in this capacity during experiments conducted in Monterey Bay, CA during the CANON/BIOSPACE experiments in October 2010. A WG platform was deployed on October 7, 2010, recovered on October 11, 2010 for a minor technical repair, redeployed on October 16, 2010, and finally recovered and the mission terminated on October 28, 2010. Apart from science data, the vehicle recorded wind speed and direction, rudder direction and its horizontal speed.

A focus of the CANON initiative is the investigation of Harmful Algal Blooms (HABs). While significant effort in understanding bloom ecology has been expended by ocean scientists and fundamental causes of biogeochemical transport of nutrient flux are understood for specific phases of bloom ecology, an understanding of the overall dynamics of phytoplankton blooms is poor to non-existent. Augmenting fixed in situ instrumentation with adaptively sampling robots performing in situ feature recognition and event response, will plug a substantial gap in our understanding of HABs and coastal ecology in general. Given the stochastic environment and the large $\left(>50 \mathrm{~km}^{2}\right)$ spatial and temporal extent of such coastal phenomena, it is necessary to ensure that robotic assets are present at the right place at the right time.

Planning vehicle missions and large-scale experiments hinging upon a temporal constraint requires a comprehensive understanding of the vehicle dynamics and its interaction with the environment. In addition to placing a vehicle in the right place at the right time, it is important to understand the capabilities of a vehicle so that appropriate missions can be planned and executed. For example, arrival at a specific location may be necessary to facilitate the interaction of a surface vessel with an underwater vehicle. Also, for persistent monitoring applications as presented in [4] and [5], it may be necessary for a vehicle to complete a surveillance circuit within a specified amount of time.

Through extensive deployments, it has been determined that the WG's forward speed depends on the amplitude of the surface waves, the overall buoyancy force provided by the float, and the glider's weight [1]. The later two in this list are determined by the vehicle design and payload, and can be considered to remain constant during a deployment. To this end, the WG's mass and buoyancy and the length of its tether have been tuned to provide excellent wave-energy propulsion performance in both energetic and calm seas alike [2]. Table I presents the observed results of the WG's forward speed for various sea states.

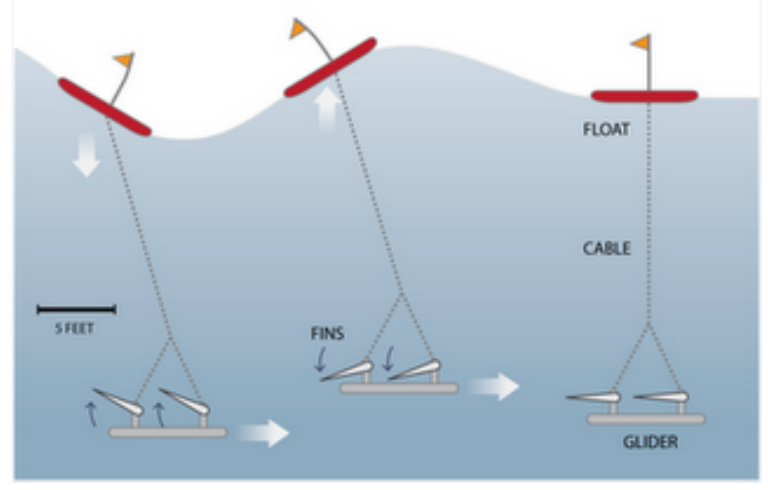

Fig. 2: The basic mechanics of locomotion for the Wave Glider.

Over long duration missions, it has been observed that the WG is able to maintain an average speed of approximately $0.8 \mathrm{~m} / \mathrm{s}$. Although this information provides a general guideline for mission planning, it is of interest to determine a more precise relationship between wave height and WG speed. Additionally, we are interested to understand if there are any other environmental factors that contribute to the operational dynamics of the vehicle. Hence, we present a system identification based on data collected during experiments conducted as part of the CANON initiative in Monterey Bay, CA in October 2010. As previously mentioned, during deployment, the WG recorded wind speed and direction, rudder direction and forward speed. Additionally, we consider ocean surface and subsurface current measurements that are provided through historical HF radar data and Acoustic Doppler Current Profiler (ADCP) data from moorings in Monterey Bay.

\section{Vehicle PARAMETERS}

The Wave Glider is a hybrid sea-surface and underwater vehicle that is composed of a submerged glider that is attached to a surface float via a tether. The WG is propelled by converting vertical wave motions into forward thrust, see Fig. 2. This type of locomotion is independent of the wave direction, as only changes in body-axis heave of the surface float result in movement of the submerged glider. The propulsion system is purely mechanical, and no electrical power generated by the propulsion mechanism. Located at $\sim 7 \mathrm{~m}$ depth, the submerged glider experiences significantly reduced forces from surface waves compared to the surface float. Hence, gliding through these relatively still waters at depth, the vertical motion of the float activates the wings, and a portion of this upward motion is converted into a forward propulsion force on the surface float. As waves pass by the surface float, the submerged glider tugs the it along a prescribed path.

Based on this two-component design, the WG is subjected to two (potentially different) dynamic and uncertain environments. The surface component is affected by wind, waves and surface currents. The subsurface component experiences a different current (possibly in magnitude and direction) than at the surface, and the effects of wind and waves are felt 


\begin{tabular}{|c|c|c|c|}
\hline Sea State & Significant Wave Height (m) & WG Speed (kts) & WG Speed (m/s) \\
\hline Flat Calm & 0 & 0 & 0 \\
\hline Sea State 0 & 0 & $0.25-0.5$ & $0.13-0.26$ \\
\hline Sea State 1 & $0-0.1$ & $0.5-1.5$ & $0.26-0.8$ \\
\hline Sea State 2 & $0.1-0.5$ & 1.25 to 2.0 & $0.64-1.03$ \\
\hline Sea State 3+ & $0.5-1.25+$ & $1.5-2.25$ & $0.8-1.16$ \\
\hline Long Mission Average & Variable & 1.5 & 0.8 \\
\hline
\end{tabular}

TABLE I: The forward speed of the Wave Glider is a function of sea state. The Wave Glider's average speed for long missions that experience a wide range of sea conditions is $1.5 \mathrm{kts}(0.8 \mathrm{~m} / \mathrm{s})$. This table is a modified version of the presentation in [1], [2].

indirectly via the Ekman spiral and cable tension from the surface component, respectively.

\section{EnVironmental and WaVe Glider Data}

We gathered a spectrum of data streams that were collected from multiple sources to supply a basis of information to create the desired model. The goal to predict WG speed offline for an upcoming experiment motivated the selection of a particular set of environmental parameters. We are interested in utilizing data sets that are readily available, and broadly applicable to a region of interest. The data chosen to analyze, and the sources of these data are presented in the following sections.

\section{A. Ocean Currents}

For the ocean currents, we are interested in both the surface current and the current at the same depth of the submerged glider $(\sim 7 \mathrm{~m})$. Surface currents were obtained by use of CODAR Ocean Sensors High Frequency Radar (HFR) measurements. These radar systems measure ocean surface currents (restricted to the upper $0.5 \mathrm{~m}$ ) using continuously transmitted/received radio waves. There are multiple radar sites around Monterey Bay, and each site produces a radial estimation of the currents, which is then combined with overlapping data from the other sites to produce a current vector map for the entire bay. Each site is networked and the data are updated hourly as interpolated (based on openboundary modal analysis or OMA) measurements output as a gridded format by the Central and Northern California Ocean Observing System (CeNCOOS) [6]. The spatial resolution of the data utilised for the analysis presented here is 1 $\mathrm{km}$. Subsurface current data were supplied by a downwardlooking ADCP attached to MBARI's MO mooring located in the center of Monterey Bay. These ADCP data are a time series recorded at the mooring location. The time series consists of speed and direction on the horizontal plane at 5 $\mathrm{m}$ depth intervals from $10 \mathrm{~m}$ to $55 \mathrm{~m}$. We used the measured currents at $10 \mathrm{~m}$ depth, as this is closest to the submerged glider section of the WG at $\sim 7 \mathrm{~m}$.

In Figs. 3 and 4, we present the distribution of the speed of the surface current as measured by the CODAR HF Radar stations, and this speed projected onto the body-frame surge axis of the WG, respectively.

\section{B. Wave Data}

The wave data used for our analysis included the significant wave height, wave peak period, and wave direction for the duration of the deployment. These data are also collected

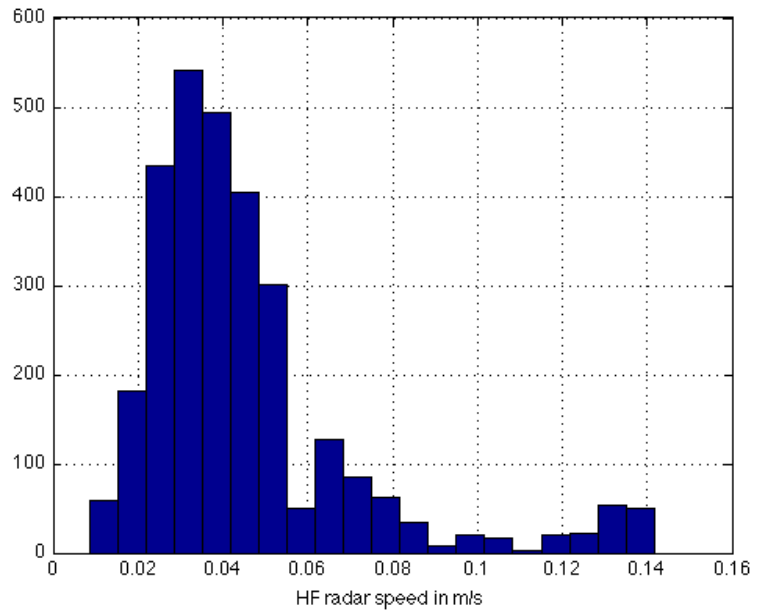

Fig. 3: The distribution of speeds of the surface current measured by the HF radar stations during the 7 -day period of analysis.

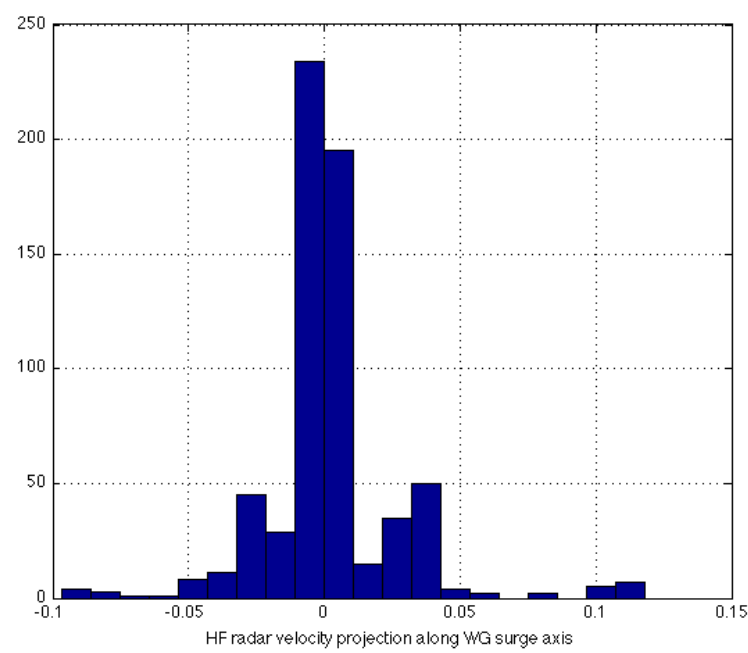

Fig. 4: The distribution of the speed of the surface current projected along the body-frame surge axis of the Wave Glider. 
by MBARI's $M O$ mooring, and made publicly available via the CenCOOS Data Portal. We use the difference between the course of the WG and the wave direction for our analysis.

\section{Wind Data}

Wind data for this analysis included both the speed and direction collected by MBARI's $M O$ mooring. Based on the height of the mooring, these data provided the best estimation of the wind characteristics occurring at the sea surface, and most likely to influence the WG. The data were obtained from the CeNCOOS Data Portal. The component of the wind velocity vector along the body-frame surge axis of the WG is used for our analysis.

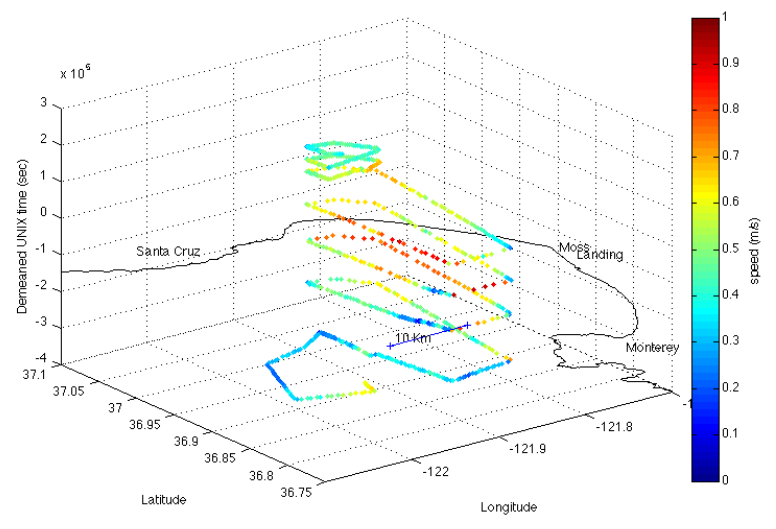

(a) Spatio-temporal view of Wave Glider path and speeds

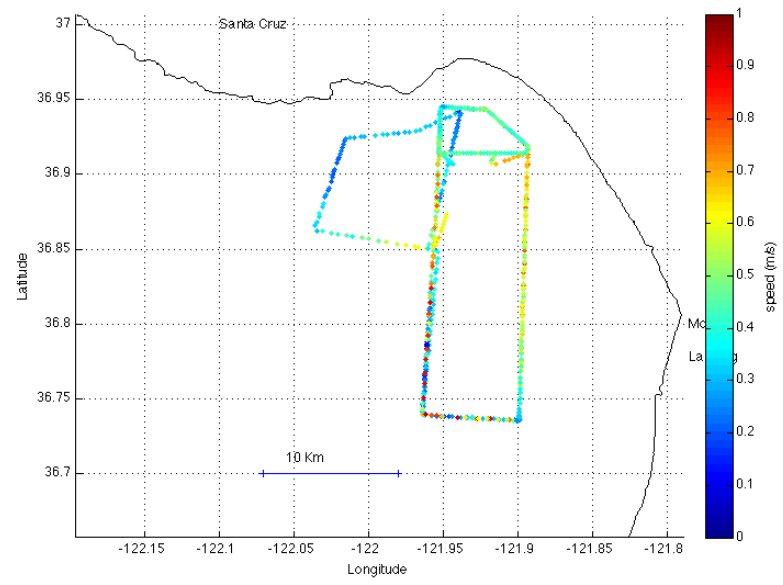

(b) Top view of Wave Glider path during the 7-day analysis period

Fig. 5: Wave Glider path and speeds during an 7-day analysis period in October 2010.

\section{Vehicle Data}

The data required from the vehicle are the current heading, commanded heading and current speed. The heading information is provided by the on-board compass, and the commanded heading is available from the mission logs of the vehicle. The speed of the WG was computed post-facto from the the GPS logged on-board the vehicle. Figure 5 presents the path followed by the WG along with the speed at each location.

\section{System Identification Method}

We combined all of the environmental parameter data and Wave Glider navigation data for a period of $\sim 7$ days, October 20, 18:30 GMT to October 27, 15:30 GMT. For this time period, we formed a conglomerate data set, consisting of 650 data points recorded at $\sim 6$ minute intervals. Figure 5 displays the path and estimated speed of the vehicle executed during this 7 day period. Our dataset consists of $N=650$ records of a $k=6$ dimensional input feature vector $\mathbf{x}=\left[x_{1} \ldots x_{6}\right]$. The elements $\left\{\mathbf{x}_{\mathbf{i}} \mid i=1, \ldots, 6\right\}$ of the feature vector are significant wave height, peak wave period, wave direction differential ${ }^{8}$, and components of currents (HF radar and $\mathrm{ADCP}$ ) and winds along body-frame surge axix of WG. These data form a $650 \times 6$ input matrix $X$. We also have 650 records of the output variable of interest; the WG speed $y^{9}$, forming an $650 \times 1$ matrix $Y$.

Our goal is to learn a linear predictive model that maps an environmental input vector $\mathbf{x}$ to predicted Wave Glider speed $y$. Hence, we want to learn the model parameters for $y=f(\mathbf{x}), \mathbf{x} \in \mathbb{R}^{6}, y \in \mathbb{R}$, where the function $f$ is defined by coefficient vector $\mathbf{w}$, such that $y=\mathbf{w}^{T} \mathbf{x}$.

The model parameters (coefficient vector $\mathbf{w}$ ) is fitted using the least squares solution to the training dataset,

$$
w=\left(X^{T} X\right)^{-1} X^{T} Y
$$

\section{RESUlts}

We partitioned our dataset into training and test subsets in the proportion of $80 \%$ and $20 \%$, respectively. This was done by selecting every fifth sample from the time series to be part of the training subset. This selection method ensured that both the training and test data sets were evenly distributed throughout the temporal scale of the data. In doing so, the training data was able to capture the variability in environmental conditions throughout the 7-day period of the experiment considered for this analysis.

Utilizing the procedure outlined in Section V, we generated a predictive model for WG speed by linearly weighting each of the six input environmental parameters. The linear regression equation for Wave Glider speed is given by,

$$
\begin{aligned}
y_{\text {sog }} & =0.1619 x_{w h}+0.0107 x_{w p p}+0.0010 x_{w d i r} \\
& +0.0023 x_{w n d}+0.0020 x_{a d c p}-0.500 x_{h f r} .
\end{aligned}
$$

Here, $y_{s o g}$ is the predicted Wave Glider speed over ground, $x_{w h}$ is the significant wave height in meters, $x_{w d i r}$ is the component of the wind speed along the WG body-frame surge axis $(\mathrm{m} / \mathrm{s}), x_{w n d}$ is the component of wind speed along the WG body-frame surge axis, $x_{a d c p}$ is the component of the ADCP measured sub-surface current (depth $=10 \mathrm{~m}$ ) along the WG body-frame surge axis, and $x_{h f r}$ is the component of the estimated surface current (from HF radar) for the location and time of the WG, along its body-frame surge axis. We remark that the dominant components are the significant wave height and the wave peak period.

Over the duration of the 7-day deployment, the WG had an average speed of $0.47 \mathrm{~m} / \mathrm{s}$, with the distribution of the speeds presented in Fig. 6.

\footnotetext{
${ }^{8}$ Difference between WG course and wave direction

${ }^{9}$ Vehicle speed was computed post-facto using logged GPS data.
} 


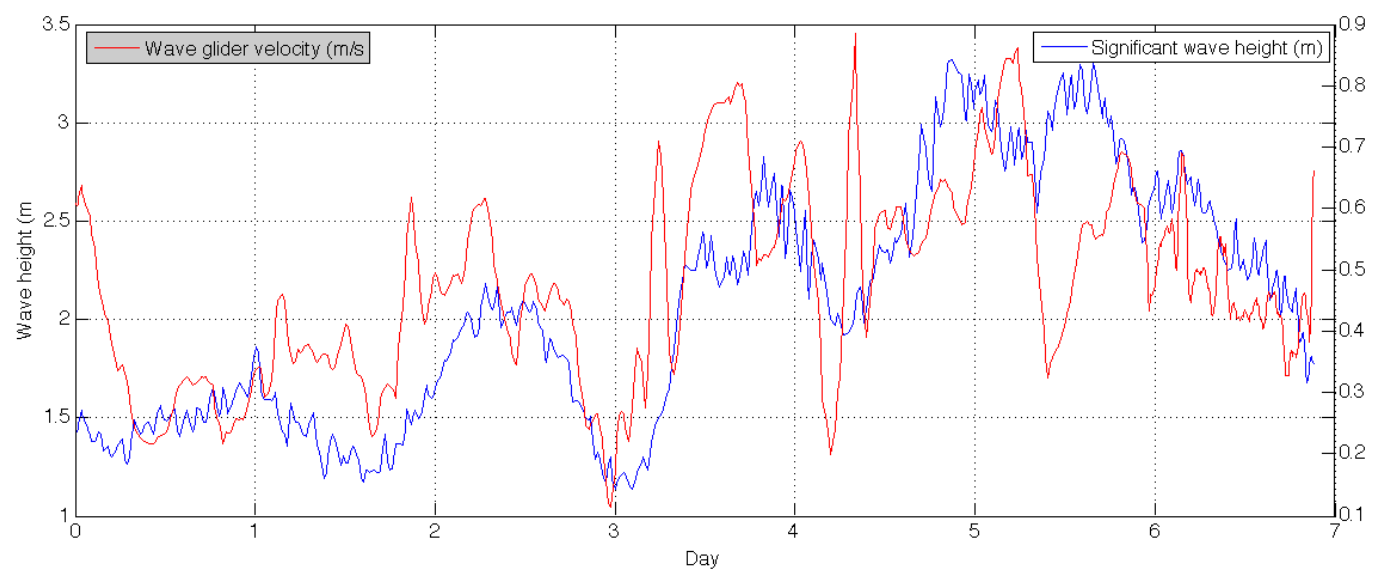

Fig. 7: Plot of Wave Glider speed vs. significant wave height.

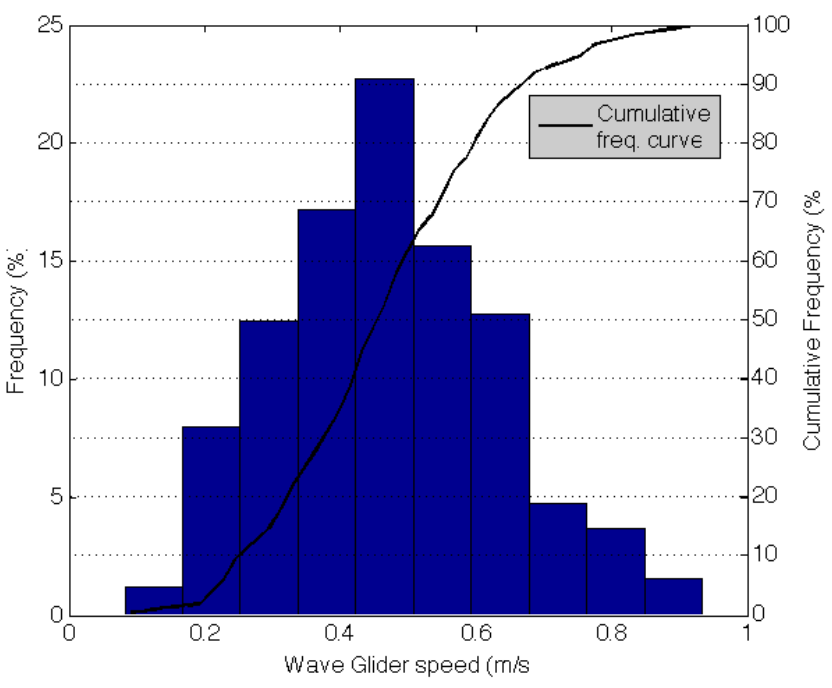

Fig. 6: The distribution of Wave Glider speeds observed during the 7-day period of analysis.

\section{A. Analysis}

The broad conclusion of our analysis shows consistency with the data presented in Table I in that the WG's speed is most correlated to the measured significant wave height. Figure 7 displays the significant wave height with the speed of the WG overlaid, and one can see a significant correlation between to two parameters. A detailed analysis shows that the correlation coefficient between the significant wave height and the WG speed is $R=0.61$. The second most influential environmental parameter on WG speed is the wave peak period. This showed a correlation coefficient of $R=0.13$. For all of the additional considered environmental parameters (wave direction, wind speed and direction, surface and subsurface currents), the maximum correlation coefficient was $R<0.05$, a full order of magnitude less than significant wave height and peak period. In Fig. 8, we show the WG speed overlaid with three of the considered environmental parameters; significant wave height, wave peak period and wave direction. In these figures, the data have been demeaned and normalized to be presented on the same axes. From these plots, we see the evident correlation between significant wave height and wave peak period, as well as the non-correlation between WG speed and wave direction as mentioned in Section III.

It is interesting to note that the average speed of the WG during the 7-day analysis period considered here was 0.47 $\mathrm{m} / \mathrm{s}$, as compared to the $0.8 \mathrm{~m} / \mathrm{s}$ average quoted in Table I and in [1] and [2]. During the 7 days considered here, the WG experienced significant wave heights between $0 \mathrm{~m}$ and $3.4 \mathrm{~m}$, as shown in Fig. 7. However, the corresponding WG speeds do not directly match those presented in Table I. Specifically, our analysis shows that the WG only achieves a speed of $\sim 0.8 \mathrm{~m} / \mathrm{s}$ with a significant wave height of $\sim 3 \mathrm{~m}$, contrary to $\sim 0.1 \mathrm{~m}$ significant wave height as presented in Table I. This difference can be explained by 1) a different deployment location, 2) additional and unknown environmental parameters affecting the WG motion, 3) the small data subset considered here is not a good representative for long-mission averages, or a combination of all three of these factors.

We expected that the surface and subsurface currents would have an impact on the WG's speed, as seen in Fig. 3. However, the mean surface current during the period of analysis had a magnitude of $0.045 \mathrm{~m} / \mathrm{s}$. As this parameter is a full order-of-magnitude less than the mean WG speed of $0.47 \mathrm{~m} / \mathrm{s}$ (Fig. 6) over the duration of the analyzed data, the impact was minimal. Additionally, we utilized an estimation of the surface currents from the land-based CODAR stations, and ADCP data from a single buoy (MBARI's MO) in Monterey Bay. Unlike measured significant wave height, which can be considered uniform over a large spatial extent [7], ocean currents have variability on relatively small spatial scales, inferring that data are required at higher spatial resolution to precisely determine any impact on the prediction of the WG velocity. Research is ongoing to investigate the utilization of ocean models to provide higher resolution information. In this case, future experiments could use the WG as an adaptively sampling sensor, sending data back to shore, which would be utilised to supplement sparse datasets that currently form the basis for predictive models. Such a cyclic process will 

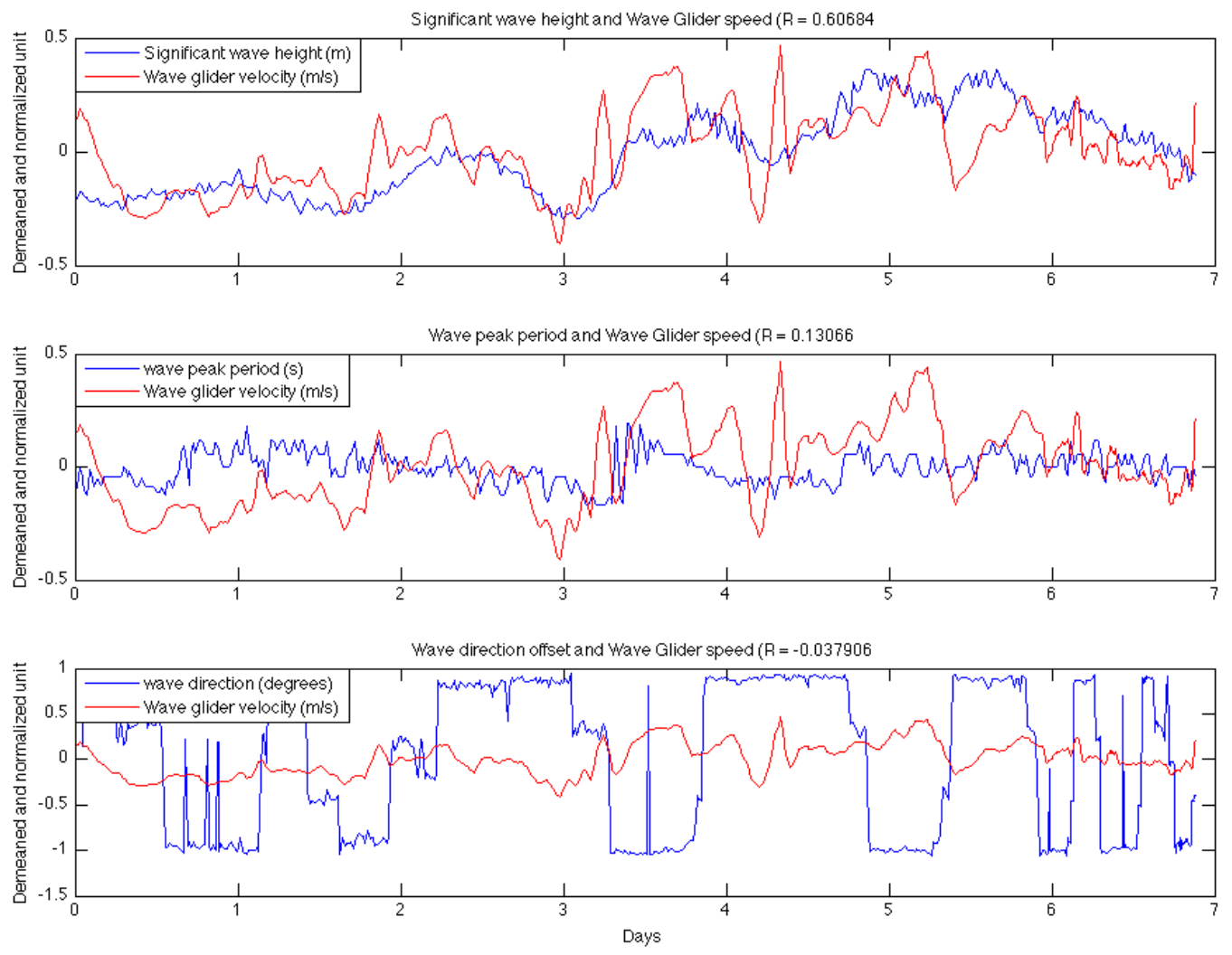

Fig. 8: Correlation between Wave Glider speeds and three of the six environmental input parameters.

likely produce better model forecasts of dynamic coastal phenomenon; an urgent need in the ocean sciences.

The training dataset was used to generate the weighting factors for each of the environmental parameters used in the linear regression model. Substituting the data points of the training dataset back into the regression model outputs the scatter-plot shown in Figure 9a. Here, the x-axis represents the true WG speed and the y-axis is the predicted WG speed given the environmental parameter inputs. These data show a general linear trend, however there are many outliers and the variability is large. Figure $9 \mathrm{~b}$ presents the results of the same process just described for the test dataset. The results here show a similar dispersement as those seen in Fig. 9a, with a basic linear trend, but many outliers and high variability.

\section{CONCLUSIONS}

In this paper, we investigated the utility of a linear regression model as a system identification tool for determining a predictive model for offline planning for the Wave Glider platform. We presented a linear regression model that predicts the vehicle speed for a desired heading, given the significant wave height, ocean surface and subsurface currents, wind speed and direction. Since the set of environmental input variables can be obtained in advance, we can implement the presented model for a specific location within a given region of operation. This can predict the WG speed over a reasonable temporal horizon. The significance is that this predictive model can be utilized to optimize a desired objective function for a given survey, e.g., ensure that prescribed waypoints are visited during certain time windows, or a closed-loop circuit is completed in an allotted time. This model is a first step towards enabling more advanced and dynamic planning of specific missions, as it provides an estimation of the WG speed based on a given path and known or predicted environmental conditions. This prior knowledge is important for ocean science applications, since obtaining a synoptic view of a feature of interest generally has a temporal, as well as a spatial dependence. Primary WG applications for robotics research are in the area of longterm, persistent control and life-long learning, thus enabling temporal sampling capabilities for a given mission is of great importance. Here, we augment the capabilities of the WG as a persistent monitoring platform by providing a tool to allow for mission planning that provides coverage, timing or performance guarantees. By providing this data-driven capability to mission planning and control, we can extend the WG's use to automated observation and inference.

The analysis presented here found that of the 6 environmental parameters examined, only 2 had significant impact upon the speed of the WG; significant wave height and wave peak period. This result agrees with the results published in [1], [2], and further defines the contribution of these factors to the overall speed of the vehicle. The presented results also show that there are other parameters that influence the 


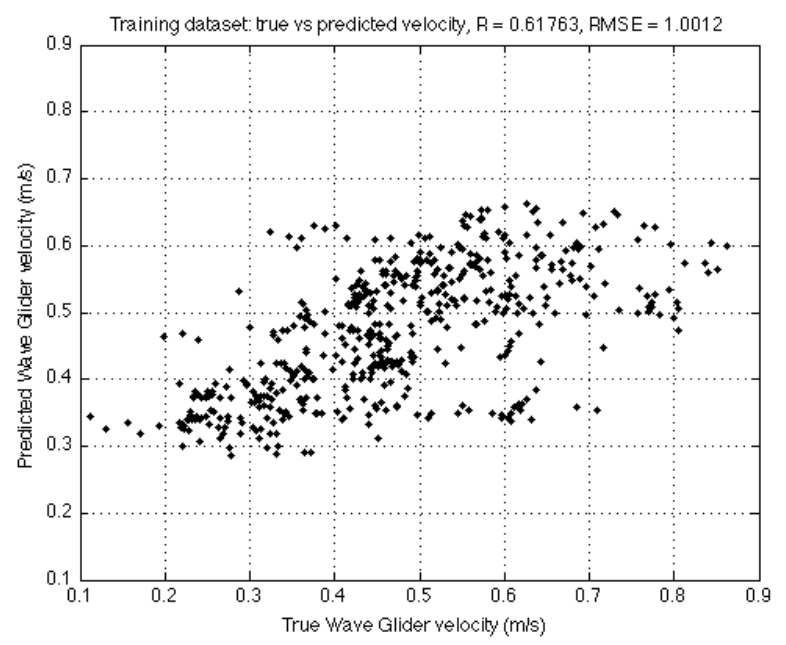

(a) True vs. predicted velocity on training dataset using learned model

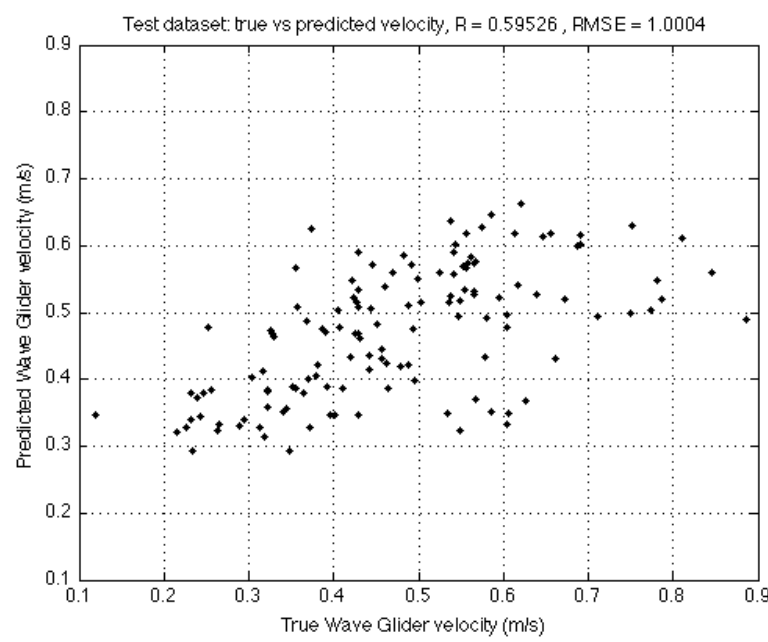

(b) True vs. predicted velocity on test dataset using learned model

Fig. 9: Model performance on training and test datasets.

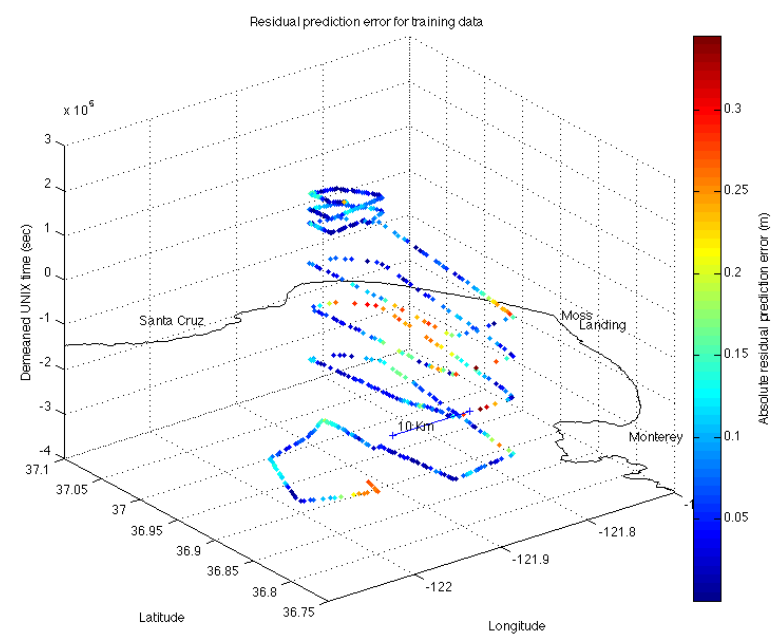

Fig. 10: The residual prediction error for the training dataset. speed of the WG that need to be examined in more detail to produce a reliable model for predicting its speed. One of these parameters is the subsurface currents that affect the submerged glider portion of the vehicle. Another parameter is the local wind near the vehicle, which may have some direct effect on the motion of the WG, but also may have a direct effect on the submerged glider via the Ekman spiral.

\section{FUTURE WORK}

The results from the analysis presented here motivate two areas of future research to further our understanding of the correlation between the WG speed and the environmental parameters that provide its propulsion.

\section{A. Subsurface Currents}

As an initial area of future work, we will examine the subsurface currents at a depth of $7 \mathrm{~m}$; approximately where the submerged glider is positioned. We will obtain highresolution current data from the Navy Coastal Ocean Model (NCOM) run by the Naval Research Laboratory for the time period of the deployment considered in this paper. This ocean model is a primitive equation, 3D, hydrostatic model that uses the MellorYamada level 2.5 turbulence closure scheme, and the Smagorinsky formulation for horizontal mixing. This model is a hierarchy of different resolution models for the West Coast of the United States [8]. Further details of this model can be found in [9] and [10].

\section{B. Non-linear Model}

In addition to acquiring more high-fidelity data streams, we plan to investigate non-parametric and non-linear regression models. Initially, we will consider utilizing a Gaussian process regression model [11]. This is a non-parametric, nonlinear, and probabilistic regression model. By use of such a model, we hypothesize that we will be able to exploit the high-dimensional input space more effectively than with a simple linear regression. An initial implementation of this technique utilizing the same data and same training dataset/test dataset split as presented earlier is displayed in Figs. 11a and 11b. The error bars displayed represent two standard deviations about the mean predictions. In these figures, we see a much higher correlation $(R)$ value as compared to Figs. 9a and 9b, but the Root Mean Square Error (RMSE) is very similar. The fact that we achieve a better correlation coefficient, yet RMSE remains comparable between the linear and non-linear models is an interesting artefact that requires further investigation.

\section{ACKNOWLEDGMENT}

This work was supported in part by the NOAA MERHAB program under grant NA05NOS4781228 and by NSF as part of the Center for Embedded Network Sensing (CENS) under grant CCR-0120778, by NSF grant CNS-1035866, by the ONR MURI program (grants N00014-09-1-1031 and N00014-08-1-0693) by the ONR SoA program and a gift from the Okawa Foundation. R.N. Smith was partially supported by the Early Career Academic Recruitment and Development (ECARD) program of the Queensland University of Technology. 


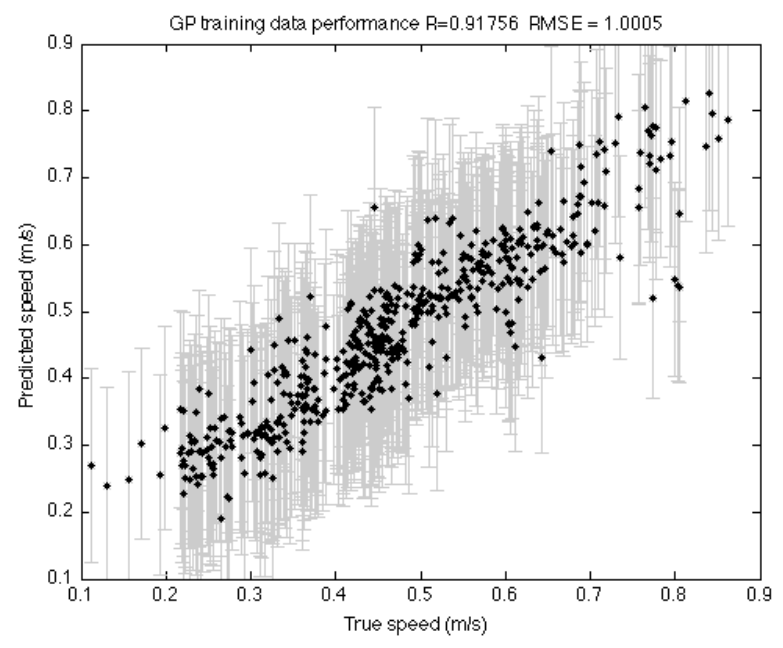

(a) True vs predicted velocity on training dataset using Gaussian Process regression model

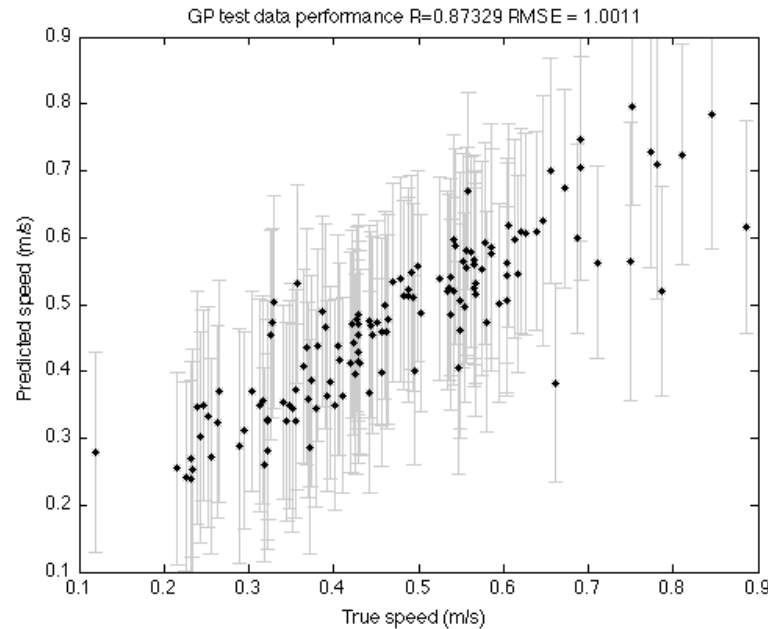

(b) True vs predicted velocity on test dataset using Gaussian Process regression model

Fig. 11: Gaussian Process regression model performance on training and test datasets. The displayed error bars are 2 standard deviations about the mean predictions.

\section{REFERENCES}

[1] R. Hine, S. Willcox, G. Hine, and T. Richardson, "The wave glider: A wave-powered autonomous marine vehicle," in OCEANS 2009, MTS/IEEE Biloxi - Marine Technology for Our Future: Global and Local Challenges, oct. 2009, pp. $1-6$.

[2] J. Manley and S. Willcox, "The wave glider: A persistent platform for ocean science," in Proceedings of the IEEE/MTS OCEANS Conference, Sydney, Australia, May 2010, pp. 1 - 5. [Online]. Available: http://ieeexplore.ieee.org/xpl/freeabs_all.jsp?arnumber $=5603614$

[3] F. Chavez, "Canon: Controlled, agile, and novel observing network," http://www.mbari.org/canon/, 2010.

[4] S. L. Smith and D. Rus, "Multi-robot monitoring in dynamic environments with guaranteed currency of observations," in IEEE Conference on Decision and Control, Atlanta, GA, December 2010.

[5] R. N. Smith, M. Schwager, S. L. Smith, B. H. Jones, D. Rus, and G. S. Sukhatme, "Persistent ocean monitoring with underwater gliders: Adapting sampling resolution," Journal of Field Robotics, 2011, to appear. [Online]. Available: http://cres.usc.edu/cgi-bin/print_ pub_details.pl?pubid=710

[6] CeNCOOS, "CeNCOOS Central \& Northern California Coastal Observing System," http://cencoos.org, 2009, viewed December 2009. [Online]. Available: http://cencoos.org
[7] R. Dean and R. Dalrymple, Water wave mechanics for engineers and scientists, ser. Advanced series on ocean engineering. World Scientific, 1991. [Online]. Available: http://books.google.com/books? $\mathrm{id}=9-\mathrm{M} 4 \mathrm{U} \backslash \operatorname{sfin} 8 \mathrm{C}$

[8] I. Shulman, J. Kindle, P. Martin, S. deRada, J. Doyle, B. Penta, S. Anderson, F. Chavez, J. Paduan, and S. Ramp, "Modeling of upwelling/relaxation events with the navy coastal ocean model," Journal of Geophysical Research, vol. 112, 2007.

[9] I. Shulman, C. Rowley, S. Anderson, S. DeRada, J. Kindle, P. Martin, J. Doyle, J. Cummings, S. Ramp, F. Chavez, D. Fratantoni, and R. Davis, "Impact of glider data assimilation on the Monterey Bay model," Deep-Sea Research II, vol. 56, pp. 188 - 198, 2009.

[10] I. Shulman, S. Anderson, C. Rowley, S. DeRada, J. Doyle, and S. Ramp, "Comparisons of upwelling and relaxation events in the Monterey Bay area," Journal of Geophysical Research, vol. 115, no. C06016, p. 12pp, 2010.

[11] C. E. Rasmussen, "Gaussian processes for machine learning." MIT Press, 2006. 